\section{Optimal Linear Estimation and Data Fusion}

Robert J. Elliott and John van der Hoek

\begin{abstract}
Optimal mean square linear estimators are determined for general uncorrelated noise. We allow the noise variance matrix in the observation process to be singular. This requires properties of generalized inverses which are developed in Section II. The proofs appear to be new. When there are two observation sequences the optimal method of recursively fusing the two is determined. We derive a new formula for the covariance of the two estimates which then provides exact dynamics for a fused estimate.
\end{abstract}

Index Terms-Data fusion, optimal linear estimation.

\section{INTRODUCTION}

Much recent work in filtering has used particle filters, which are really Monte Carlo simulations; see, for example, [3]. However, the case of singular noise still gives problems. We believe that the use of the weak generalized inverse in filtering with singular noise is new.

Fusion problems have been extensively studied; see [2]. For the fusion of information from two linear filters, ad-hoc combinations are still often proposed as in [6]. In Section IV, we obtain the recursion for the covariance of the noise in two linear filters. This is then used to obtain the optimal fused estimate. This result appears to be new.

We consider a signal process $\left\{X_{k}\right\}$ with linear dynamics

$$
X_{k+1}=F_{k} X_{k}+G_{k} W_{k+1} .
$$

Here, the noise terms $W_{k+1}$ are a sequence of uncorrelated, zero mean, (not necessarily Gaussian), random variables.

The $X$ process is not observed directly, but through two noisy observation processes ${ }^{1} Y,{ }^{2} Y$ with

$$
\begin{aligned}
{ }^{1} Y_{k} & ={ }^{1} H_{k} X_{k}+{ }^{1} V_{k} \\
{ }^{2} Y_{k} & ={ }^{2} H_{k} X_{k}+{ }^{2} V_{k} .
\end{aligned}
$$

Again, the noise terms ${ }^{1} V_{k},{ }^{2} V_{k}$ are sequences of uncorrelated, zero mean (not necessarily Gaussian) random variables.

We suppose variance matrices related to the observation processes $Y$ are possibly singular. This requires the use of a weak notion of generalized inverse which is discussed in Section II.

The optimal linear mean square filter is reviewed in Section III and the fusion of two such filters for observation sequences $\left\{{ }^{1} Y_{k}\right\}$ and $\left\{{ }^{2} Y_{k}\right\}$ is given in Section IV.

\section{GENERALIZED INVERSES}

Consider a probability space $(\Omega, \mathcal{F}, \mathbb{P})$. Vectors will be considered as column vectors. $*$ will denote the transpose. $L^{2}\left(\Omega, R^{n}\right)$ will denote the set of random variables $Y: \Omega \rightarrow R^{n}$ such that $E\left[|Y|^{2}\right]=\int_{\Omega} Y^{*} Y d \mathbb{P}<\infty$. This is a Hilbert space with inner product $\int_{\Omega} X^{*} Y d \mathbb{P}$.

Suppose $P$ denotes an expectation, or a conditional expectation operator.

Manuscript received April 14, 2003; revised August 12, 2004 and November 22, 2005. Recommended by Associate Editor L. Gerencser.

R. J. Elliott is with the Haskayne School of Business, University of Calgary, Calgary, AB T2N 1N4 Canada (e-mail: relliott@ucalgary.ca).

J. van der Hoek are with the Department of Applied Mathematics, University of Adelaide, Adelaide 5005, Australia (e-mail: jvanderhmaths.adelaide.edu.au).

Digital Object Identifier 10.1109/TAC.2006.872768
Definition II.1: A generalized inverse of a matrix $A$ is a matrix $A^{\#}$ such that

$$
A A^{\#} A=A \text {. }
$$

If $A$ is nonsingular then $A^{\#}=A^{-1}$. However, generalized inverses need not be unique. The Moore-Penrose generalized inverse of $A$ is a matrix $A^{+}$such that

$$
\begin{aligned}
A A^{+} A & =A \quad A^{+} A A^{+}=A^{+} \\
\left(A A^{+}\right)^{*} & =A A^{+} \text {and }\left(A^{+} A\right)^{*}=A^{+} A .
\end{aligned}
$$

The Moore-Penrose generalized inverse is unique.

For details on generalized inverses, see [1], [4], and [5].

We will work with generalized inverses satisfying (1).

Notation II.2: Suppose $X \in L^{2}\left(\Omega, R^{m}\right), Y \in L^{2}\left(\Omega, R^{n}\right)$. Consider the covariance matrices

$$
\begin{aligned}
& \Delta_{X Y}=E\left[(X-P(X))(Y-P(Y))^{*}\right] \\
& \Delta_{Y Y}=E\left[(Y-P(Y))(Y-P(Y))^{*}\right] .
\end{aligned}
$$

Suppose $\Delta_{Y Y}^{\#}$ is any generalized inverse of $\Delta_{Y Y}$.

Notation II.3: Write $\nu_{Y}=\Delta_{Y Y}^{\#}(Y-P(Y))$. Note $P\left(\nu_{Y}\right)=0$.

Linear algebra establishes the following results.

Lemma II.4: $Y=P(Y)+\Delta_{Y Y} \nu_{Y}$ a.s.

Lemma II.5: $\Delta_{X Y} \Delta_{Y Y}^{\#} \Delta_{Y Y}=\Delta_{X Y}$.

Lemma II.6: $\Delta_{Y Y} \Delta_{Y Y}^{\#}(Y-P(Y))=Y-P(Y)$.

Lemma II.7: Suppose $X \in L^{2}\left(\Omega, R^{m}\right), Y \in L^{2}\left(\Omega, R^{n}\right)$ and $\Delta_{Y Y}^{\#}$ is a generalized inverse of $\Delta_{Y Y}$. Then, if

$$
\begin{aligned}
C & =-\Delta_{X Y} \Delta_{Y Y}^{\#} \\
\text { and } Z & =X-P(X)+C(Y-P(Y))
\end{aligned}
$$

we have $E\left[Z(Y-P(Y))^{*}\right]=\Delta_{Z Y}=0 \in R^{m \times n}$.

Lemma II.8: For random variables $X: \Omega \rightarrow R^{m}, Y: \Omega \rightarrow R^{n}$

$E\left[(X-P(X)-B(Y-P(Y)))^{*}\right.$

$$
(X-P(X)-B(Y-P(Y)))]
$$

is minimized when $B=\Delta_{X Y} \Delta_{Y Y}^{\#}$ where $\Delta_{Y Y}^{\#}$ is any generalized inverse of $\Delta_{Y Y}$, that is, any matrix such that

$$
\Delta_{Y Y} \Delta_{Y Y}^{\#} \Delta_{Y Y}=\Delta_{Y Y} .
$$

Remark II.9: From Lemma II.8, we see, with this $C, Z=X-$ $P(X)-\Delta_{X Y} \Delta_{Y Y}^{\#}(Y-P(Y))$ is the projection error when projecting $X-P(X)$ onto $Y-P(Y)$.

Corollary II.10: Suppose $\widetilde{\Delta}_{Y Y}^{\#}$ is another generalized inverse of $\Delta_{Y Y}$ and $\widetilde{Z}=X-P(X)-\Delta_{X Y} \widetilde{\Delta}_{Y Y}^{\#}(Y-P(Y))$. Then, $E[(Z-$ $\left.\widetilde{Z})(Z-\widetilde{Z})^{*}\right]=0$.

In particular, $Z=\widetilde{Z}$ a.e.

Notation II.11: $E\left[Z(Y-P(Y))^{*}\right]=0$ implies that the projection of $Z$ on $Y$ is 0 and we write $P_{Y}(Z)=0$. As $P_{Y}$ is to be linear, we define

$$
P_{Y}(Z)=0=P_{Y}(X)-P(X)-\Delta_{X Y} \Delta_{Y Y}^{\#}(Y-P(Y)) .
$$

That is, for $X \in L^{2}\left(\Omega, R^{m}\right), Y \in L^{2}\left(\Omega, R^{n}\right)$

$$
P_{Y}(X)=P(X)+\Delta_{X Y} \Delta_{Y Y}^{\#}(Y-P(Y)) .
$$

Remark II.12: $P_{Y}(X)$ gives the projection of $X$ onto $Y$ and

$$
P_{Y}(X)=E[X]+\Sigma_{X Y} \Sigma_{Y Y}^{\#}(Y-E[Y]) .
$$

In the sequel, the expectation is denoted either by $E$ or $P$. If, further, $X$ and $Y$ are Gaussian this expression gives the conditional expected value of $X$ given $Y$. 
Consider now the case where $Y=\left[\begin{array}{l}Y_{1} \\ Y_{2}\end{array}\right]$ with $Y_{1} \in L^{2}\left(\Omega, R^{n_{1}}\right)$, $Y_{2} \in L^{2}\left(\Omega, R^{n_{2}}\right)$. Suppose, as before, $X \in L^{2}\left(\Omega, R^{m}\right)$. Then

$$
\begin{aligned}
\Delta_{Y Y} & =E\left[(Y-P(Y))(Y-P(Y))^{*}\right] \\
& =\left[\begin{array}{ll}
\Delta_{Y_{1} Y_{1}} & \Delta_{Y_{1} Y_{2}} \\
\Delta Y_{2} Y_{1} & \Delta Y_{2} Y_{2}
\end{array}\right]=\left[\begin{array}{ll}
A & B \\
C & D
\end{array}\right]
\end{aligned}
$$

say, where $\Delta_{Y_{i} Y_{j}}=E\left[\left(Y_{i}-P\left(Y_{i}\right)\right)\left(Y_{j}-P\left(Y_{j}\right)\right)^{*}\right]$.

Lemma II.13: Write $S_{A}=D-C A^{\#} B$

$$
=\Delta_{Y_{2} Y_{2}}-\Delta_{Y_{2} Y_{1}} \Delta_{Y_{1} Y_{1}}^{\#} \Delta_{Y_{1} Y_{2}} \text {. }
$$

Then, $S_{A}$ is nonnegative and symmetric. Write $S_{D}=A-B D^{\#} C$

$$
=\Delta_{Y_{1} Y_{1}}-\Delta_{Y_{1} Y_{2}} \Delta_{Y_{2} Y_{2}}^{\#} \Delta_{Y_{2} Y_{1}} \text {. }
$$

Then, $S_{D}$ is nonnegative and symmetric.

Lemma II.14: $A A^{\#} B=B$ and $C A^{\#} A=C$.

The following result is noted in Rohde [4].

Lemma II.15: If $E=A^{\#}+A^{\#} B S_{A}^{\#} C A^{\#}$

$$
\begin{aligned}
& F=-A^{\#} B S_{A}^{\#} \\
& G=-S_{A}^{\#} C A^{\#} \\
& H=S_{A}^{\#}
\end{aligned}
$$

then $\left[\begin{array}{ll}E & F \\ G & H\end{array}\right]$ is a generalized inverse of $\left[\begin{array}{cc}A & B \\ C & D\end{array}\right]$.

\section{OPTIMUM LINEAR FILTERS}

Without the usual assumption that the observation noise is nonsingular we now derive the optimum linear filter for linear dynamics in the state and observation processes. The square integrable noise terms are assumed uncorrelated. When the noise terms are Gaussian we obtain the optimum least-square filter.

Model III.1: Suppose $\left\{X_{k}\right\}, k=0,1,2, \ldots$ is a sequence of square integrable, $R^{m}$-valued random variables such that

$$
X_{k+1}=F_{k} X_{k}+G_{k} W_{k+1} .
$$

Here, $\left\{W_{k}\right\}$ is a sequence of uncorrelated random variables such that for $k, \ell=0,1, \ldots$

$$
\begin{aligned}
E\left[W_{k}\right] & =0 \\
E\left[W_{k} W_{\ell}^{*}\right] & =Q_{k} \delta_{k \ell} \\
\text { and } E\left[X_{0} W_{k}^{*}\right] & =0 .
\end{aligned}
$$

Here, $\delta_{k \ell}=0$ if $k \neq \ell$ and $\delta_{k k}=1$.

The observations are given by a sequence $\left\{Y_{k}\right\}, k=0,1,2, \ldots$ of square integrable random variables with values in $R^{n}$. Further

$$
Y_{k}=H_{k} X_{k}+V_{k} .
$$

$\left\{V_{k}\right\}$ is a sequence of uncorrelated random variables such that for $k$, $\ell=0,1, \ldots$

$$
\begin{aligned}
E\left[V_{k}\right] & =0 \\
E\left[V_{k} V_{\ell}^{*}\right] & =R_{k} \delta_{k \ell} \\
\text { and } E\left[X_{0} V_{k}^{*}\right] & =0 .
\end{aligned}
$$

Further, $E\left[W_{k} V_{\ell}^{*}\right]=0$ for all $k, \ell$. The $H_{k}, F_{k}, G_{k}$ are time varying matrices of appropriate dimension.

Suppose $X_{0}$ has mean $m_{0}$ and variance $\Sigma_{0}$. Define $P\left(X_{0}\right)=$ $E\left[X_{0}\right]=m_{0}$ so $P\left(Y_{0}\right)=E\left[Y_{0}\right]=E\left[H_{0} X_{0}+V_{0}\right]=H_{0} m_{0}$. From (2)

$$
\begin{aligned}
P_{Y_{0}}\left(X_{0}\right) & =E\left[X_{0}\right]+\Sigma_{X_{0} Y_{0}} \Sigma_{Y_{0} Y_{0}}^{\#}\left(Y_{0}-E\left[Y_{0}\right]\right) \\
& =m_{0}+\Sigma_{X_{0} Y_{0}} \Sigma_{Y_{0} Y_{0}}^{\#}\left(Y_{0}-H_{0} m_{0}\right) .
\end{aligned}
$$

Note $\Sigma_{X_{0} Y_{0}}=\Sigma_{0} H_{0}^{*}$ and $\Sigma_{Y_{0} Y_{0}}=H_{0} \Sigma_{0} H_{0}^{*}+R_{0}$. Write $P_{0}$ for $P_{Y_{0}}$.

Recurrence III.2: Suppose $P_{0}\left(X_{0}\right), \ldots, P_{k}\left(X_{k}\right)$ have been obtained and the next observation $Y_{k+1}$ is received

$$
Y_{k+1}=H_{k+1} X_{k+1}+V_{k+1} \text {. }
$$

We wish to determine the projection $P_{k+1}\left(X_{k+1}\right)$ of $X_{k+1}$ onto the space spanned by $Y_{0}, Y_{1}, \ldots, Y_{k+1}$.

Lemma III.3: $P_{k+1}\left(X_{k+1}\right)=F_{k} P_{k}\left(X_{k}\right)$

$$
+\Delta_{X Y}^{k+1}\left(\Delta_{Y Y}^{k+1}\right)^{\#}\left(Y_{k+1}-H_{k+1} F_{k} P_{k}\left(X_{k}\right)\right) .
$$

Proof: Consider $Z_{k+1}=X_{k+1}-P_{k}\left(X_{k+1}\right)+C_{k+1}\left(Y_{k+1}-\right.$ $\left.P_{k}\left(Y_{k+1}\right)\right)$. We wish to choose $C_{k+1}$ so that

$$
E\left[Z_{k+1}\left(Y_{k+1}-P_{k}\left(Y_{k+1}\right)\right)^{*}\right]=0 .
$$

This is the case if

where

$$
C_{k+1}=-\Delta_{X Y}^{k+1}\left(\Delta_{Y Y}^{k+1}\right)^{\#}
$$

$$
\Delta_{X Y}^{k+1}=E\left[\left(X_{k+1}-P_{k}\left(X_{k+1}\right)\right)\left(Y_{k+1}-P_{k}\left(Y_{k+1}\right)\right)^{*}\right]
$$

and

$$
\Delta_{Y Y}^{k+1}=E\left[\left(Y_{k+1}-P_{k}\left(Y_{k+1}\right)\right)\left(Y_{k+1}-P_{k}\left(Y_{k+1}\right)\right)^{*}\right] .
$$

$\left(\Delta_{Y Y}^{k+1}\right)^{\#}$ is a generalized inverse of $\Delta_{Y Y}^{k+1}$. With this choice for $C_{k+1} P_{k+1}\left(Z_{k+1}\right)=0$ so

$$
\begin{aligned}
P_{k+1}\left(X_{k+1}\right)= & P_{k}\left(X_{k+1}\right) \\
& +\Delta_{X Y}^{k+1}\left(\Delta_{Y Y}^{k+1}\right)^{\#}\left(Y_{k+1}-P_{k}\left(Y_{k+1}\right)\right) \\
= & F_{k} P_{k}\left(X_{k}\right) \\
& +\Delta_{X Y}^{k+1}\left(\Delta_{Y Y}^{k+1}\right)^{\#}\left(Y_{k+1}-H_{k+1} F_{k} P_{k}\left(X_{k}\right)\right)
\end{aligned}
$$

as $P_{k}\left(W_{k+1}\right)=0$ and $P_{k}\left(V_{k+1}\right)=0$.

$P_{k+1}\left(X_{k+1}\right)$ gives the best linear least squares estimate of $X_{k+1}$ given $Y_{0}, Y_{1}, \ldots, Y_{k+1} . P_{k}\left(X_{k}\right)$ has already been determined and the new information is provided by $Y_{k+1}$.

We finally show how $C_{k+1}$ is updated. Write

$$
\Delta_{X X}^{k \mid k}=E\left[\left(X_{k}-P_{k}\left(X_{k}\right)\right)\left(X_{k}-P_{k}\left(X_{k}\right)\right)^{*}\right]
$$

for the error covariance at time $k$.

Lemma 3.3: $C_{k+1}=-\Delta_{X Y}^{k+1}\left(\Delta_{Y Y}^{k+1}\right)^{\#}$ where

$$
\begin{aligned}
\Delta_{X Y}^{k+1} & =\Delta_{X X}^{k+1} H_{k+1}^{*} \\
\Delta_{Y Y}^{k+1} & =H_{k+1} \Delta_{X X}^{k+1} H_{k+1}^{*}+R_{k+1} \\
\Delta_{X X}^{k+1} & =F_{k} \Delta_{X X}^{k \mid k} F_{k}^{*}+G_{k} Q_{k+1} G_{k}^{*} \\
\Delta_{X X}^{k+1 \mid k+1} & =\Delta_{X X}^{k+1}-\Delta_{X Y}^{k+1}\left(\Delta_{Y Y}^{k+1}\right)^{\#}\left(\Delta_{X Y}^{k+1}\right)^{*} .
\end{aligned}
$$

\section{Fusion OF OPTIMAL LINEAR FILTERS}

Suppose as in Section III $\left\{X_{k}\right\}, k=0,1,2, \ldots$ is a signal process with dynamics

$$
X_{k+1}=F_{k} X_{k}+G_{k} W_{k+1} .
$$

However, suppose now we have two observation processes $\left\{{ }^{1} Y_{k}\right\}$, $\left\{{ }^{2} Y_{k}\right\}, k=0,1,2, \ldots$. Write

$$
Y_{k}=\left(\begin{array}{c}
{ }^{1} Y_{k} \\
{ }^{2} Y_{k}
\end{array}\right) \text {. }
$$


We suppose ${ }^{i} Y_{k}={ }^{i} H_{k} X_{k}+{ }^{i} V_{k}, i=1,2, k=0,1,2, \ldots$ The $\left\{{ }^{i} V_{k}\right\}$ are sequences of zero-mean uncorrelated noises with

$$
\begin{aligned}
E\left[{ }^{i} V_{k}\right] & =0 \\
E\left[{ }^{i} V_{k}{ }^{i} V_{\ell}^{*}\right] & ={ }^{i} R_{k} \delta_{k \ell} \\
\text { and } E\left[X_{0}{ }^{i} V_{k}^{*}\right] & =0, \quad i=1,2 .
\end{aligned}
$$

Further

$$
E\left[{ }^{1} V_{k}^{2} V_{\ell}^{*}\right]=\Gamma_{k} \delta_{k \ell}
$$

However, we assume the $\Gamma_{k}$ are not known a priori. They are to be modeled and estimated from the observations. This could be because the observer has some control over ${ }^{1} Y$ and ${ }^{2} Y$ separately but not jointly.

Notation IV.1: ${ }^{12} P_{k}$ denotes the projection onto $\left\{Y_{0}, Y_{1}, \ldots, Y_{k}\right\}$. For $i=1,2,{ }^{i} P_{k}$ denotes the projection onto $\left\{{ }^{i} Y_{0},{ }^{i} Y_{1}, \ldots,{ }^{i} Y_{k}\right\}$. Write

$$
\begin{aligned}
H_{k}= & \left({ }^{1} H_{k}^{*},{ }^{2} H_{k}^{*}\right)^{*} \\
V_{k}=( & \left({ }^{1} V_{k}^{*},{ }^{2} V_{k}^{*}\right)^{*} \\
\text { so that } Y_{k}= & H_{k} X_{k}+V_{k} \\
{ }^{12} \Delta_{X Y}^{k+1}=E & {\left[\left(X_{k+1}-{ }^{12} P_{k}\left(X_{k+1}\right)\right)\right.} \\
& \left.\times\left(Y_{k+1}-{ }^{12} P_{k}\left(Y_{k+1}\right)\right)^{*}\right] \\
=E & {\left[\left(X_{k+1}-{ }^{12} P_{k}\left(X_{k+1}\right)\right)\right.} \\
& \times\left({ }^{1} Y_{k+1}-{ }^{12} P_{k}\left({ }^{1} Y_{k+1}\right)\right)^{*} \\
& \left(X_{k+1}-{ }^{12} P_{k}\left(X_{k+1}\right)\right) \\
& \left.\times\left({ }^{2} Y_{k+1}-{ }^{12} P_{k}\left({ }^{2} Y_{k+1}\right)\right)^{*}\right] \\
& {\left[\left(Y_{k+1}-{ }^{12} P_{k}\left(Y_{k+1}\right)\right)\right.} \\
& \left.\times\left(Y_{k+1}-{ }^{12} P_{k}\left(Y_{k+1}\right)\right)^{*}\right] .
\end{aligned}
$$

Problem IV.2: With the combined observation process $\left\{Y_{k}\right\}$ the result of Section III gives

$$
\begin{aligned}
{ }^{12} P_{k+1}\left(X_{k+1}\right)= & { }^{12} P_{k}\left(X_{k+1}\right) \\
& -{ }^{12} C_{k+1}\left(Y_{k+1}-{ }^{12} P_{k}\left(Y_{k+1}\right)\right) \\
= & F_{k}{ }^{12} P_{k}\left(X_{k}\right) \\
& -{ }^{12} C_{k+1} \\
& \times\left(Y_{k+1}-H_{k+1} F_{k}{ }^{12} P_{k}\left(X_{k}\right)\right)
\end{aligned}
$$

with ${ }^{12} C_{k+1}=-{ }^{12} \Delta_{X Y}^{k+1}\left({ }^{12} \Delta_{Y Y}^{k+1}\right) \#$ for some generalized inverse $\left({ }^{12} \Delta_{Y Y}^{k+1}\right)^{\#}$.

The results of Section III give update formulas for $i=1,2$

$$
\begin{aligned}
{ }^{i} P_{k+1}\left(X_{k+1}\right)=F_{k}{ }^{i} P_{k}\left(X_{k}\right) & \\
& -{ }^{i} C_{k+1}\left({ }^{i} Y_{k+1}-{ }^{i} H_{k+1} F_{k}{ }^{i} P_{k}\left(X_{k}\right)\right)
\end{aligned}
$$

with

$$
\begin{aligned}
{ }^{i} C_{k+1} & =-{ }^{i} \Delta_{X Y}^{k+1}\left({ }^{i} \Delta_{Y Y}^{k+1}\right)^{\#} \\
{ }^{i} \Delta_{X Y}^{k+1} & =E\left[\left(X_{k+1}-{ }^{i} P_{k}\left(X_{k+1}\right)\right)\left({ }^{i} Y_{k+1}-{ }^{i} P_{k}\left({ }^{i} Y_{k+1}\right)\right)^{*}\right]
\end{aligned}
$$
and

$$
{ }^{i} \Delta_{Y Y}^{k+1}=E\left[\left(Y_{i+1}-{ }^{i} P_{k}\left(Y_{k+1}\right)\right)\left(Y_{k+1}-{ }^{i} P_{k}\left(Y_{k+1}\right)\right)^{*}\right] .
$$

The fusion problem is to determine a recursive expression for ${ }^{12} P_{k}\left(X_{k}\right)$ in terms of ${ }^{1} Y_{k},{ }^{2} Y_{k},{ }^{1} P_{k}\left(X_{k}\right)$, and ${ }^{2} P_{k}\left(X_{k}\right)$.
Suppose ${ }^{12} P_{k}\left(X_{k}\right)$ is known and we wish to determine ${ }^{12} P_{k+1}\left(X_{k+1}\right)$. From (7), we see we need only determine

$$
{ }^{12} C_{k+1}=-{ }^{12} \Delta_{X Y}^{k+1}\left({ }^{12} \Delta_{Y Y}^{k+1}\right) \# .
$$

Now ${ }^{12} P_{k}\left(X_{k+1}\right)=F_{k}{ }^{12} P_{k}\left(X_{k}\right)$ is known and

$$
\begin{aligned}
{ }^{12} P_{k}\left(Y_{k+1}\right)= & \left(\left({ }^{12} P_{k}\left({ }^{1} Y_{k+1}\right)\right)^{*},\left({ }^{12} P_{k}\left({ }^{2} Y_{k+1}\right)\right)^{*}\right)^{*} \\
= & \left(\left({ }^{1} H_{k+1} F_{k}{ }^{12} P_{k}\left(X_{k}\right)\right)^{*}\right. \\
& \left.\left({ }^{2} H_{k+1} F_{k}{ }^{12} P_{k}\left(X_{k}\right)\right)^{*}\right)^{*}
\end{aligned}
$$

is known. Therefore, for $i=1,2$

$$
\begin{aligned}
& E\left[\left(X_{k+1}-{ }^{12} P_{k}\left(X_{k+1}\right)\right)\left({ }^{i} Y_{k+1}-{ }^{12} P_{k}\left({ }^{i} Y_{k+1}\right)\right)^{*}\right] \\
&= E\left[\left(F_{k}\left(X_{k}-{ }^{12} P_{k}\left(X_{k}\right)\right)+G_{k} W_{k+1}\right)\right) \\
& \times\left(\left(X_{k}-{ }^{12} P_{k}\left(X_{k}\right)\right){ }^{*} F_{k}^{* i} H_{k+1}^{*}\right. \\
&\left.\left.+W_{k+1}^{*} G_{k}^{* i} H_{k+1}^{*}+{ }^{i} V_{k+1}^{*}\right)\right] \\
&= F_{k}{ }^{12} \Delta_{X X}^{k \mid k} F_{k}^{* i} H_{k+1}^{*}+G_{k} Q_{k+1} G_{k}^{* i} H_{k+1}^{*} .
\end{aligned}
$$

From (6)

$$
\begin{aligned}
{ }^{12} \Delta_{X Y}^{k+1}=\left[F_{k}{ }^{12} \Delta_{X X}^{k \mid k} F_{k}^{* 1} H_{k+1}^{*}+G_{k} Q_{k+1} G_{k}^{* 1} H_{k+1}^{*}\right. \\
\left.F_{k}{ }^{12} \Delta_{X X}^{k \mid k} F_{k}^{* 2} H_{k+1}^{*}+G_{k} Q_{k+1} G_{k}^{* 2} H_{k+1}^{*}\right]
\end{aligned}
$$

and ${ }^{12} \Delta_{X Y}^{k+1}$ is known if

$$
{ }^{12} \Delta_{X X}^{k \mid k}=E\left[\left(X_{k}-{ }^{12} P_{k}\left(X_{k}\right)\right)\left(X_{k}-{ }^{12} P_{k}\left(X_{k}\right)\right)^{*}\right]
$$

is known.

$$
\begin{aligned}
{ }^{12} \Delta_{X X}^{k+1}= & E\left[\left(X_{k+1}-{ }^{12} P_{k}\left(X_{k+1}\right)\right)\right. \\
& \left.\times\left(X_{k+1}-{ }^{12} P_{k}\left(X_{k+1}\right)\right)^{*}\right] \\
= & F_{k}{ }^{12} \Delta_{X X}^{k \mid k} F_{k}^{*}+G_{k} Q_{k+1} G_{k}^{*}
\end{aligned}
$$

and is known if ${ }^{12} \Delta_{X X}^{k \mid k}$ is known. As in Section III, the update for ${ }^{12} \Delta_{X X}^{k+1 \mid k+1}$ is given by

$$
{ }^{12} \Delta_{X X}^{k+1 \mid k+1}={ }^{12} \Delta_{X X}^{k+1}-{ }^{12} \Delta_{X Y}^{k+1}\left({ }^{12} \Delta_{Y Y}^{k+1}\right)^{\#}\left({ }^{12} \Delta_{X Y}^{k+1}\right)^{*} .
$$

The only remaining term is

$$
\begin{aligned}
{ }^{12} \Delta_{Y Y}^{k+1}= & E\left[\left(Y_{k+1}-{ }^{12} P_{k}\left(Y_{k+1}\right)\right)\left(Y_{k+1}-{ }^{12} P_{k}\left(Y_{k+1}\right)\right)^{*}\right] \\
= & E\left[\left[\begin{array}{l}
{ }^{1} Y_{k+1}-{ }^{12} P_{k}\left({ }^{1} Y_{k+1}\right) \\
{ }^{2} Y_{k+1}-{ }^{12} P_{k}\left({ }^{2} Y_{k+1}\right)
\end{array}\right]\right. \\
& \times\left[{ }^{1} Y_{k+1}-{ }^{12} P_{k}\left({ }^{1} Y_{k+1}\right)^{*}\right. \\
& \left.\left.{ }^{2} Y_{k+1}-{ }^{12} P_{k}\left({ }^{2} Y_{k+1}\right)^{*}\right]\right] \\
= & {\left.\left[\begin{array}{ll}
A & B \\
C & D
\end{array}\right], \quad \text { say, (dropping the } k+1 \text { suffix }\right) . }
\end{aligned}
$$


Now

$$
\begin{aligned}
A=E & {\left[\left({ }^{1} Y_{k+1}-{ }^{12} P_{k}\left({ }^{1} Y_{k+1}\right)\right)\left({ }^{1} Y_{k+1}-{ }^{12} P_{k}\left({ }^{1} Y_{k+1}\right)\right)\right] } \\
=E & {\left[\left({ }^{1} H_{k+1} F_{k}\left(X_{k}-{ }^{12} P_{k}\left(X_{k}\right)\right)\right.\right.} \\
& \left.+{ }^{1} H_{k+1} G_{k} W_{k+1}+{ }^{1} V_{k+1}\right) \\
& \times\left({ }^{1} H_{k+1} F_{k}\left(X_{k}-{ }^{12} P_{k}\left(X_{k}\right)\right)\right. \\
& \left.\left.\left.\quad+{ }^{1} H_{k+1} G_{k} W_{k+1}+{ }^{1} V_{k+1}\right)\right)^{*}\right] \\
={ }^{1} H_{k+1} F_{k}{ }^{12} \Delta_{X X}^{k \mid k} F_{k}^{* 1} H_{k+1}^{*} & +{ }^{1} H_{k+1} G_{k} Q_{k+1} G_{k}^{* 1} H_{k+1}^{*}+{ }^{1} R_{k} .
\end{aligned}
$$

Similarly

$$
\begin{aligned}
D={ }^{2} H_{k+1} F_{k}{ }^{12} \Delta_{X X}^{k \mid k} F_{k}^{* 2} H_{k+1}^{*} & \\
& +{ }^{2} H_{k+1} G_{k} Q_{k+1} G_{k}^{* 2} H_{k+1}^{*}+{ }^{2} R_{k} .
\end{aligned}
$$

The interesting term is

$$
\begin{aligned}
C^{*}=B & \\
=E & {\left[\left({ }^{1} H_{k+1} F_{k}\left(X_{k}-{ }^{12} P_{k}\left(X_{k}\right)\right)\right.\right.} \\
& \left.+{ }^{1} H_{k+1} G_{k} W_{k+1}+{ }^{1} V_{k+1}\right) \\
& \times\left({ }^{2} H_{k+1} F_{k}\left(X_{k}-{ }^{12} P_{k}\left(X_{k}\right)\right)\right. \\
& \left.\left.\quad+{ }^{2} H_{k+1} G_{k} W_{k+1}+{ }^{2} V_{k+1}\right)\right] \\
= & { }^{1} H_{k+1} F_{k}{ }^{12} \Delta^{k \mid k} X X F_{k}^{* 2} H_{k+1}^{*} \\
& +{ }^{1} H_{k+1} G_{k} Q_{k+1} G_{k}^{* 2} H_{k+1}^{*}+E\left[{ }^{1} V_{k+1}{ }^{2} V_{k+1}^{*}\right] .
\end{aligned}
$$

Remarks IV.3: If $\Gamma_{k+1}=E\left[{ }^{1} V_{k+1}{ }^{2} V_{k+1}^{*}\right]$ is known then $B$ and $C$ are known. Consequently, a generalized inverse $\left({ }^{12} \Delta_{Y Y}^{k+1}\right){ }^{\#}$ of ${ }^{12} \Delta_{Y Y}^{k+1}=\left[\begin{array}{cc}A & B \\ C & D\end{array}\right]$ is given by Lemma II.17 and the update

$$
{ }^{12} \Delta_{X X}^{k+1 \mid k+1}={ }^{12} \Delta_{X X}^{k+1}-{ }^{12} \Delta_{X Y}^{k+1}\left({ }^{12} \Delta_{Y Y}^{k+1}\right){ }^{\#}\left({ }^{12} \Delta_{X Y}^{k+1}\right){ }^{*}
$$

is determined, as is

$$
{ }^{12} C_{k+1}=-{ }^{12} \Delta_{X Y}^{k+1}\left({ }^{12} \Delta_{Y Y}^{k+1}\right) \# .
$$

Consequently, ${ }^{12} P_{k+1}\left(X_{k+1}\right)$ is then given.

In summary, given ${ }^{12} \Delta_{X X}^{k \mid k}$ we can compute ${ }^{12} \Delta_{X Y}^{k+1}$ and ${ }^{12} \Delta_{X X}^{k+1}$. If we also know $\Gamma_{k}$ we can find

$$
{ }^{12} \Delta_{Y Y}^{k+1} \text {, giving }\left({ }^{12} \Delta_{Y Y}^{k+1}\right)^{\#}
$$

and then ${ }^{12} C_{k+1}$. The unknown quantity is the covariance $\Gamma_{k+1}=$ $E\left[{ }^{1} V_{k+1}{ }^{2} V_{k+1}^{*}\right]$ between the noise terms in the two observations.

We shall investigate the relation between $\Gamma_{k+1}$ and the covariance of

$$
\left({ }^{1} Y_{k+1}-{ }^{1} P_{k}\left({ }^{1} Y_{k+1}\right)\right) \text { and }\left({ }^{2} Y_{k+1}-{ }^{2} P_{k}\left({ }^{2} Y_{k+1}\right)\right) .
$$

Notation IV.4: Write $\Lambda_{k+1}=E\left[\left({ }^{1} Y_{k+1}-\right.\right.$ $\left.\left.{ }^{1} P_{k}\left({ }^{1} Y_{k+1}\right)\right)\left({ }^{2} Y_{k+1}-{ }^{2} P_{k}\left({ }^{2} Y_{k+1}\right)\right)^{*}\right]$. In Section V, we discuss how $\Lambda$ might be described.

Write

$$
\begin{aligned}
M_{k+1}=E\left[\left(X_{k+1}-{ }^{1} P_{k+1}\left(X_{k+1}\right)\right)\right. & \\
& \left.\times\left(X_{k+1}-{ }^{2} P_{k+1}\left(X_{k+1}\right)\right)^{*}\right] .
\end{aligned}
$$

Recall that for $i=1,2$ from Section III

$$
{ }^{i} P_{k+1}\left(X_{k+1}\right)=F_{k}{ }^{i} P_{k}\left(X_{k}\right)-{ }^{i} C_{k+1}\left({ }^{i} Y_{k+1}-{ }^{i} P_{k}\left({ }^{i} Y_{k+1}\right)\right)
$$

with ${ }^{i} C_{k+1}=-{ }^{i} \Delta_{X Y}^{k+1}\left({ }^{i} \Delta_{Y Y}^{k+1}\right)^{\#}$.

The recurrences for ${ }^{i} C_{k+1}$ are known for $i=1,2$. We can then show the following.

Lemma IV.5: The recurrence for $M$ is given by

$$
\begin{aligned}
M_{k+1}= & F_{k} M_{k} F_{k}^{*}+{ }^{1} C_{k+1}{ }^{1} H_{k+1} F_{k} M_{k} F_{k}^{*} \\
& +F_{k} M_{k} F_{k}^{* 2} H_{k+1}^{*} C_{k+1}^{*}+G_{k} Q_{k+1} G_{k}^{*} \\
& +{ }^{1} C_{k+1} \Lambda_{k+1}{ }^{2} C_{k+1} .
\end{aligned}
$$

Lemma IV.6: From the definition given in Notation IV.4, it is easily checked that

$$
\Lambda_{k+1}={ }^{1} H_{k+1} M_{k+1}{ }^{2} H_{k+1}^{*}+E\left[{ }^{1} V_{k+1}{ }^{2} V_{k+1}^{*}\right] .
$$

Remarks IV.7: If $\Lambda_{k+1}$ is given and $M_{k}$ is known then the result of Lemma IV.5 gives $M_{k+1}$.

Lemma IV.6 then gives $E\left[{ }^{1} V_{k+1}{ }^{2} V_{k+1}^{*}\right]$. The terms $B$ and $C$ are then determined allowing the recursion to proceed, as described in Remarks IV.3.

To initialize the process, consider

$$
\begin{aligned}
& M_{0}=E\left[\left(X_{0}-{ }^{1} P_{0}\left(X_{0}\right)\right)\left(X_{0}-{ }^{2} P_{0}\left(X_{0}\right)\right)^{*}\right] \\
& =E\left[\left(X_{0}-E\left[X_{0}\right]-\Sigma_{X_{0}{ }^{1} Y_{0}} \Sigma_{1_{Y_{0}}{ }^{1} Y_{0}}^{\#}\left({ }^{1} Y_{0}-E\left[{ }^{1} Y_{0}\right]\right)\right)\right. \\
& \times\left(X_{0}-E\left[X_{0}\right]-\Sigma_{X_{0}{ }^{2} Y_{0}}\right. \\
& \left.\left.\times \Sigma_{2 Y_{0} Y_{0}}^{\#}\left({ }^{2} Y_{0}-E\left[{ }^{2} Y_{0}\right]\right)\right)^{*}\right]
\end{aligned}
$$

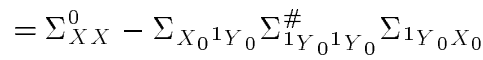

$$
\begin{aligned}
& -\Sigma_{X_{0} Y_{0} Y_{2}} \Sigma_{2 Y_{0} Y_{0}}^{\#} \Sigma_{2 Y_{0} X} \\
& +\Sigma_{X_{0} 1_{Y_{0}}} \Sigma_{1 Y_{0}{ }^{1} Y_{0}}^{\#} \Sigma_{1_{Y_{0}} Y_{Y_{0}}} \Sigma_{2 Y_{0}{ }^{2} Y_{0}}^{\#} \Sigma_{2 Y_{0} X_{0}} .
\end{aligned}
$$

This can be computed if we assume

$$
\Sigma_{1_{Y_{0} Y_{0}}}=\Lambda_{0}
$$

is known. In fact

$$
\begin{aligned}
\Sigma_{X_{0}}{ }^{i} Y_{0} & =\Sigma_{X X}^{0}{ }^{i} H_{0}^{*}, \quad i=1,2 \\
\Sigma_{{ }^{i} Y_{0}}{ }^{i} Y_{0} & ={ }^{i} H_{0} \Sigma_{X X}^{0}{ }^{i} H_{0}^{*}+{ }^{i} R_{0} \\
\text { where }{ }^{i} R_{0} & =E\left[{ }^{i} V_{0}{ }^{i} V_{0}^{*}\right] .
\end{aligned}
$$

Knowing these quantities $M_{0}$ is determined.

If $\Lambda_{k}$ is specified at each time $M_{k}$ and the $E\left[{ }^{1} V_{k}{ }^{2} V_{k}^{*}\right]$ are known then the recursion is complete.

\section{REFERENCES}

[1] S. Barnett, Matrices in Control Theory: With Applications to Linear Programming. London, U.K.: Van Nostrand, 1971, pp. 221-221.

[2] D. L. Hall and J. Llina, Handbook of Multisensor Data Fusion. Boca Raton, FL: CRC Press, 2001.

[3] B. Ristic, S.. Arulampalam, and N. Gordon, Beyond the Kalman Filter: Particle Filters for Tracking Applications. Norwood, MA: Artech House, 2004.

[4] C. A. Rohde, "Generalized inverses of partitioned matrices," J. Soc. Ind. App. Math., vol. 13, pp. 1033-1035, 1965.

[5] - "Some results on generalized inverses," SIAM Rev., vol. 8, pp. 201-205, 1966.

[6] J. Uhlmann, "Covariance consistency methods for fault tolerant distributed data fusion," Inform. Fusion, pp. 201-205, 2003. 